\title{
COLISIÓN Y ARMONIZACIÓN DE PRINCIPIOS HUMANITARIOS
}

\author{
COLLISION AND HARMONIZATION OF HUMANITARIAN \\ PRINCIPLES
}

BENJAMÍN SÁNCHEZ COBO*

\section{Resumen}

Este artículo explora las formas en que las organizaciones humanitarias definen, incorporan y movilizan orientaciones éticas globales en sus esquemas comprensivos, marcos normativos, y estrategias de acción. Como caso de estudio se abordan las tensiones en la adopción de principios humanitarios por parte de las organizaciones que conforman la Red de Ayuda Humanitaria Chilena. Se identifican tres fórmulas generales de armonización: sincretismo normativo, interpretación minimalista de la independencia financiera, y adopción de los principios en estructuras periféricas y equivalentes operativos. Por último, se plantea que adherir al principio de neutralidad presenta ventajas y desventajas para el sistema humanitario, por lo que la armonización del régimen humanitario es tan necesaria como su dispersión normativa.

\section{Palabras Clave}

Movimiento humanitario, armonización normativa, principios humanitarios, sociología jurídica.

Artículo recibido para su evaluación el 22 de mayo de 2021, y aprobado para su publicación el 25 de julio de 2021.

*Sociólogo, Magíster en Métodos para la Investigación Social. Asistente de investigación de los proyectos Fondecyt Regular \#1190265 y Fondecyt de iniciación \#11190483.

Santiago de Chile. Email: bscobo@gmail.com. 


\begin{abstract}
This article explores the ways in which humanitarian organizations define, incorporate, and mobilize global ethical guidelines in their comprehensive and normative frameworks, and strategies for action. The organizations that assemble the Chilean Humanitarian Aid Network and their tensions in the adoption of humanitarian principles are used as a case study. Three general harmonization formulas are identified: normative syncretism, minimalist interpretation of financial independence, and adoption of the principles in peripheral structures and operational equivalents. Finally, it is suggested that adhering to the principle of neutrality presents advantages and disadvantages for the humanitarian system, hence the harmonization of the humanitarian regime is not necessarily preferable to its normative dispersion.
\end{abstract}

\title{
Keywords
}

Humanitarian movement, normative harmonization, humanitarian principles, sociology of law.

\section{INTRODUCCIÓN.}

Este artículo explora las diversas formas en que las organizaciones humanitarias definen, incorporan y movilizan orientaciones éticas globales en sus esquemas comprensivos, marcos normativos, y estrategias de acción. Como caso de estudio, se abordan las tensiones implicadas en la adopción de principios humanitarios transnacionales por parte de las organizaciones de la sociedad civil (OSC) que conforman la Red de Ayuda Humanitaria Chilena (Rahch), poniendo énfasis en las fórmulas mediante las cuales estos principios son interpretados, adecuados, y en definitiva, contextualizados a su realidad local.

En el ámbito del derecho internacional, las organizaciones humanitarias han sido reguladas desde 1949 por el Derecho Internacional Humanitario (DIH). Sin embargo, este marco jurídico surge principalmente para regular el uso de la fuerza entre Estados, razón por la cual remite escasamente al quehacer de las organizaciones humanitarias privadas, lo cual ha motivado que la mayor parte de su regulación haya sido elaborada por sí mismas a través de manuales, protocolos o estatutos de adscripción voluntaria 
(soft law) ${ }^{1}$. El movimiento humanitario internacional se ha autorregulado oficialmente por cuatro principios éticos: humanidad, imparcialidad, neutralidad, e independencia, moldeando una matriz de pensamiento que representa el núcleo ético del humanitarismo "clásico", cuya formalización puede ser rastreada en la creación de estos principios por el Comité Internacional de la Cruz Roja (ICRC) en 1965.

Los cuatro principios humanitarios universales representan un tipo de humanitarismo que surge estableciendo una frontera tajante con el sistema político, lo cual queda resguardado por el principio de imparcialidad y se refuerza por los principios de neutralidad e independencia. Sin embargo, a lo largo de la década de 1990 surgió un modo de comprender el humanitarismo que entrega mayor protagonismo a los derechos humanos, al desarrollo humano y a la persecución de la imparcialidad como justicia social, lo cual tensiona el principio de humanidad. Este modelo fue denominado como "neo-humanitarismo", el cual ha entrado en contradicción con el llamado humanitarismo "clásico" u ortodoxo². Así, la tensión entre el humanitarismo clásico y el neo-humanitarismo se sumó a contradicciones sostenidas entre principios humanitarios universales con particularismos culturales o religiosos ${ }^{3}$, y a potenciales contradicciones entre los mismos principios. Considerando la fragmentación del humanitarismo en modelos con distintos imperativos éticos y lógicas de acción, se abre la interrogante sobre las dinámicas mediante las cuales el movimiento humanitario entrega consistencia a sus fundamentos éticos a escala local. Desde el punto de vista normativo, esta pregunta se traduce en cómo los principios humanitarios universales son armonizados con los preceptos éticos de organizaciones humanitarias concretas ${ }^{4}$.

1 LEEBAW, B.: "The politics of impartial activism: humanitarianism and human rights". Perspectives on Politics, Vol. 5, N², 2007. p. 225.

2 FOX, F.: "New humanitarianism: does it provide a moral banner for the 21st century?". En: Disasters, Vol. 25, N 4 , 2001. p. 277.

3 BENAVIDES, L.: El régimen internacional del "nuevo humanitarismo". Disertación doctoral, Universidad Autónoma de Madrid, Grupo de Estudios de Relaciones Internacionales (GERI), 2009. p. 121.

4 El concepto de armonización, más precisamente, refiere a la definición de estándares comunes entre dos o más cuerpos normativos para alcanzar consistencia en las prácticas que prescriben. La armonización no busca crear una semejanza total entre los objetos regulados, sino alcanzar equivalentes funcionales entre distintos perímetros normativos, por medio de ejes mínimos de convergencia que posibilitan una coordinación fluida entre las distintas instituciones, organizaciones y actores. Para ello, se eliminan o atenúan las incongruencias, incompatibilidades y contradicciones que obstruyen la integración, a la vez que se cubren lagunas o vacíos regulatorios. Por ende, la armonización no 
Para identificar los focos de colisión y las fórmulas de armonización implicadas en la interacción de las organizaciones humanitarias con los principios humanitarios clásicos, se aplicaron entrevistas en profundidad a los representantes de las OSC miembros de la Rahch, junto con una revisión de documentos internos de cada entidad. Posteriormente, se aplicó un análisis de contenido cualitativo a los relatos, con el fin de dilucidar el sentido, las normas y las prácticas asociadas a la adopción (o rechazo) de los principios humanitarios.

Los hallazgos permiten iluminar las dinámicas mediante las cuales el humanitarismo expresa sus principios éticos universales a escala local, y la consecuencia de estos procesos en distintos modos de interpretarlos, adaptarlos y practicarlos. Más específicamente, se identifican tres fórmulas generales de armonización: (a) sincretismo normativo, mediante el cual se articula la ética humanitaria con elementos propios de distintos sistemas de pensamiento y de la cultura de cada organización; (b) interpretación minimalista de la independencia financiera, referente a la adaptación y flexibilización de dicho principio a las necesidades que enfrentan las OSC en un entorno institucional económicamente adverso; y (c) adopción de los principios en estructuras periféricas y equivalentes operativos, como método para adherir a los principios humanitarios sin incorporarlos en el núcleo ético de la organización. Por último, se plantea que el actuar en conformidad con el principio de neutralidad presenta simultáneamente ventajas y desventajas para el despliegue humanitario, por lo que la armonización interna del régimen humanitario puede no ser un objetivo deseable per se.

Los resultados de esta reflexión permiten identificar focos de convergencia y complementariedad extrapolables a otros regímenes sectorial de soft-law, facilitando la delimitación de expectativas, intereses y un lenguaje común a los múltiples actores y organizaciones involucradas, lo cual incrementa sus capacidades de sincronización en contextos de planificación y despliegue. Además, entrega luces sobre cómo influyen las características particulares de determinados entornos institucionales en la capacidad de los actores locales para adherir a normas transnacionales.

necesariamente requiere la definición de una única autoridad normativa. Véase: LERNER, P.: "Sobre armonización, derecho comparado y la relación entre ambos". En: Boletín mexicano de derecho comparado, Vol. 37, N¹11, 2004. p. 921. 


\section{CONCEPTUALIZANDO PRINCIPIOS ÉTICOS: LÓGICAS, OBJETOS Y TENSIONES DE LA ACCIÓN HUMANITARIA.}

En 1965 el ICRC proclama los principios fundamentales que guían la acción humanitaria, con especial énfasis en impedir la intromisión de intereses y fuerzas políticas que, según esta organización, podían contaminar el interés último de la entidad, orientado a prevenir y aliviar el sufrimiento protegiendo la salud, la vida y la dignidad de los seres humanos ${ }^{5}$. Además, el ICRC tenía como objetivo entregar consistencia a la multiplicidad de filiales en las que se despliega la institución, con rasgos culturales e idiosincráticos que podían debilitar la identidad del Movimiento de la Cruz Roja y la Medialuna Roja ${ }^{6}$. Los principios humanitarios clásicos no tienen igual relevancia, distinguiéndose entre los sustantivos (humanidad e imparcialidad) y los derivados ${ }^{7}$ (independencia y neutralidad), descritos a continuación.

Principio de Humanidad: "Se debe aliviar el sufrimiento humano dondequiera que sea necesario. El objetivo de la acción humanitaria es proteger la vida y la salud y garantizar el respeto hacia el ser humano."8. Para comprender los fundamentos del principio de humanidad, se requiere distinguir entre una serie de conceptos interrelacionados: la actitud humana (describe a alguien "benevolente" con sus pares), lo humanitario (como cualquier acción beneficiosa para los seres humanos), y el humanitarismo (doctrina que persigue la felicidad de la especie humana) ${ }^{9}$. En un sentido biológico puro, se plantea que la asistencia mutua proviene del instinto de preservación, que contribuye a la supervivencia de nuestra especie.

Principio de Imparcialidad: "La acción humanitaria debe atender a las personas únicamente en función de sus necesidades, dando prioridad a los casos de necesidad más urgentes, con independencia de cualquier otro criterio que suponga discriminación por nacionalidad, raza, sexo, creencia religiosa, clase social o ideología política, sin ningún tipo de

$5 \quad$ PICTET, J.: The Fundamental Principles of the Red Cross: Commentary By Jean Pictet. International Federation of Red Cross and Red Crescent Societies, 1979. p. 6.

6 Ibídem, p. 7.

7 Es decir, permiten traducir los principios sustantivos en formas más concretas de acción.

8 CHS ALLIANCE, GROUP URD, PROYECTO ESFERA: La Norma Humanitaria Esencial en materia de calidad y rendición de cuentas (Core Humanitarian Standard), 2015. p. 8.

9 PICTET, J.: The Fundamental Principles of the Red Cross: Commentary By Jean Pictet. International Federation of Red Cross and Red Crescent Societies, 1979. p. 13. 
distinción adversa." ${ }^{10}$. Este principio compuesto por los criterios de nodiscriminación y proporcionalidad. La no-discriminación surge como derivación del principio de igualdad entre las personas, cuya base está en el reconocimiento de los humanos como seres equivalentes en su dignidad ${ }^{11}$. Por otro lado, el ideal de proporcionalidad (o equidad) estipula que la ayuda humanitaria debe ser suministrada en proporción con los sufrimientos, priorizando las necesidades más urgentes ${ }^{12}$.

Principio de Independencia: "La acción humanitaria debe ser independiente de objetivos políticos, económicos, militares $u$ otros que cualquier actor pueda tener con respecto a zonas donde se presta asistencia humanitaria." ${ }^{\prime 3}$. Si el principio de imparcialidad busca liberar a los actores humanitarios de sus coacciones internas, el principio de independencia tiene como objetivo impedir las coacciones externas. Las decisiones tomadas por la Cruz Roja deben estar exentas de influencias políticas, económicas o religiosas, a fin de garantizar su autonomía operativa y deliberativa. Además, Schenkenberg ${ }^{14}$ señala que pueden reconocerse tres tipos de independencia: política (autonomía decisional); financiera (automantención económica); y operativa (recursos tecnológicos y logísticos) ${ }^{15}$.

Principio de Neutralidad: "Los actores humanitarios deben abstenerse de tomar partido en las hostilidades e involucrarse en las controversias de orden político, racial, religioso o ideológico." ${ }^{\text {.16 }}$. El principio de neutralidad corresponde al fundamento ético más controvertido y que mayor confusión

10 CHS ALliANCE, GROUP URD, PROYECTO ESFERA: La Norma Humanitaria Esencial en materia de calidad y rendición de cuentas (Core Humanitarian Standard), 2015. p. 8.

11 LEEBAW, B.: "The politics of impartial activism: humanitarianism and human rights”. Perspectives on Politics, Vol. 5, N`2, 2007. 227.

12 PICTET, J.: The Fundamental Principles of the Red Cross: Commentary By Jean Pictet. International Federation of Red Cross and Red Crescent Societies, 1979. p. 27.

13 CHS ALLIANCE, GROUP URD, PROYECTO ESFERA: La Norma Humanitaria Esencial en materia de calidad y rendición de cuentas (Core Humanitarian Standard), 2015. p. 8.

14 SCHENKENBERG, E.: "Coming clean on neutrality and independence: The need to assess the application of humanitarian principles". En: International Review of the Red Cross, Vol. 97, Nº897-898, 2016. p. 308.

15 La independencia operativa y la independencia financiera refieren al mismo objeto, en la medida que los recursos técnicos o humanos dependen particularmente del financiamiento con el que cuenta cada organización. Por lo mismo, el presente estudio solo considera la independencia política y la financiera.

16 CHS ALLIANCE, GROUP URD, PROYECTO ESFERA: La Norma Humanitaria Esencial en materia de calidad y rendición de cuentas (Core Humanitarian Standard), 2015. p. 8. 
ha suscitado a lo largo de la historia. El sentido del término es particularmente negativo, en la medida que alguien neutral es quien no se identifica con bando alguno ${ }^{17}$. En este sentido, la neutralidad presupone la existencia de -al menos- dos personas o grupos en oposición, y la abstención por parte de un tercero a formar parte o a favorecer a alguna de las dos facciones.

Estos principios trascendieron las fronteras del Movimiento de la Cruz Roja y de la Medialuna Roja, formando eventualmente parte del núcleo ético de la comunidad humanitaria mundial.

\section{DISCREPANCIAS Y TRANSFORMACIONES DE LA ÉTICA HUMANITARIA}

Entre 2013 y 2014 el ICRC decidió evaluar su propia práctica de los principios por medio de un estudio llevado a cabo en siete delegaciones, las cuales operaban en diversos contextos y con configuraciones muy distintas. El estudio reveló diversas contradicciones entre los mismos principios $^{18}$, dado que si bien la noción antropológica subyacente a los principios representa valores transversales múltiples religiones y culturas, pueden ser incompatibles con algunas cosmovisiones. Por ejemplo, según Gordon y Donini ${ }^{19}$ los principios humanitarios son una producción cultural particular de las naciones del atlántico norte, lo cual coincide con el hecho de que la mayor parte de las organizaciones humanitarias internacionales provienen de Estados Unidos o Europa. Asimismo, $\mathrm{Kraft}^{20}$ describe cómo las organizaciones evangélicas que entregan asistencia humanitaria en Siria interpretan el principio de imparcialidad de un modo congruente con sus valores religiosos, aunque en tensión con el principio secular de universalidad que promueve un trato igualitario para creyentes y no-creyentes.

17 SCHENKENBERG, E.: "Coming clean on neutrality and independence: The need to assess the application of humanitarian principles". En: International Review of the Red Cross, Vol. 97, N897-898, 2016. p. 304.

18 LABBÉ, J., DAUDIN, P.: "Applying the humanitarian principles: Reflecting on the experience of the International Committee of the Red Cross". En: International Review of the Red Cross, Vol. 97, N897/898, 2016. p. 198.

19 GORDON, S., DONINI, A.: "Romancing principles and human rights: Are humanitarian principles salvageable?". En: International Review of the Red Cross, Vol. 97, N (897898), 2015. p. 78.

20 KRAFT, K.: "Faith and impartiality in humanitarian response: Lessons from Lebanese evangelical churches providing food aid”. En: International Review of the Red Cross, Vol. 97, N897-898, 2016. p. 411. 
Sumado a lo anterior, en la década de 1970 el movimiento humanitario experimentó una escisión en su institucionalidad ética con la fundación de Médecins Sans Frontières (Médicos sin Fronteras) por Bernard Kouchner, ex-trabajador de la Cruz Roja. Esta situación estimuló el surgimiento de dos visiones contrapuestas sobre el alcance de la ayuda humanitaria: una que aboga por una separación tajante entre la política y lo humanitario a fin de evitar la contaminación de este último espacio; y otra que aboga por la transformación de las estructuras económicas, políticas y culturales para conceder a los individuos paz y progreso, atacando no solo los síntomas del sufrimiento humano sino también sus causas ${ }^{21}$. En la década de los noventa, la postura más "incisiva" del humanitarismo comenzó a masificar su presencia dentro del movimiento, reforzando una manera de entender el humanitarismo que entra en tensión con su concepción ortodoxa.

$\mathrm{Al}$ respecto, Fiona Fox ${ }^{22}$ fue la primera persona en acuñar el concepto de "neo-humanitarismo", como una corriente de pensamiento ecléctica que ofrecería una alternativa para relegitimar a las organizaciones humanitarias. El neo-humanitarismo se caracterizaría por asumir una postura política que atenta implícita o explícitamente contra el principio de neutralidad, el cual es comprendido como una barrera para la protección de los derechos humanos, para la promoción del desarrollo humano, e inclusive para el cumplimiento del mismo imperativo humanitario ${ }^{23}$.

Según Leebaw ${ }^{24}$ el DIH no busca terminar con las guerras, sino "domesticarlas" para minimizar la muerte y el sufrimiento. Por ende, se abandonan las pretensiones de juzgar la causa de los conflictos bélicos (jus ad bellum) para limitar las conductas mediante las cuales se desarrolla (jus in bello). Dicho de otro modo, al menos en su raíz, el humanitarismo no pretende promover el pacifismo sino la regulación de los medios a través del cual se despliegan los combates. El movimiento de los derechos humanos, en cambio, concibe la imparcialidad como un instrumento al servicio de la acción política, encaminada a transformar la sociedad de modo permanente ${ }^{25}$.

21 BRAUMAN, R.: "Médecins Sans Frontières and the ICRC: matters of principle". En: International Review of the Red Cross, Vol. 94, N888, 2012. p. 1533.

22 FOX, F.: "New humanitarianism: does it provide a moral banner for the 21 st century?". En: Disasters, Vol. 25, ํ4, 2001. p. 275.

23 MILLS, K.: "Neo-humanitarianism: the role of international humanitarian norms and organizations in contemporary conflict". En: Global Governance, Vol. 11, 2005. p. 166.

24 LEEBAW, B.: "The politics of impartial activism: humanitarianism and human rights". Perspectives on Politics, Vol. 5, N², 2007. p. 225.

25 Ibídem, p. 226. 
Por otra parte, la incorporación del enfoque de desarrollo humano en el movimiento humanitario plantea dificultades similares a las que conlleva la adopción del enfoque de derechos humanos, aunque el foco en este caso está puesto en los efectos de la construcción de capacidades (capacitybuilding) en el principio de neutralidad, el cual dista de la "compasión minimalista" inherente al humanitarismo clásico ${ }^{26}$. La superación del asistencialismo humanitario implica enfatizar el trabajo de prevención y preparación ex-ante, por medio de metodologías participativas a nivel de comunidades locales. Resulta evidente en este punto la contradicción entre la extensión temporal a corto plazo de la ayuda humanitaria y las transformaciones más profundas y a largo plazo que propone el desarrollo humano, como sucede también con el enfoque de derechos humanos. Así, se replantea la interrogante en torno a cómo un el movimiento humanitario es capaz de definir sus contornos éticos en virtud de su alta heterogeneidad interna y potenciales focos de colisión.

\section{METODOLOGÍA.}

Se empleó la entrevista en profundidad semi-estructurada a profesionales como técnica de producción de datos, en base a una pauta de preguntas descriptivas, estructurales y de contraste, aunque se privilegió estas últimas para que el informante clave comparara y evaluara de manera crítica diferentes posturas, estándares y prácticas. Se motivó a que los relatos reflejasen la cultura organizacional en la que se enmarca cada entrevistado, de tal modo que el sujeto distinguiese entre su opinión personal y la postura de la entidad a la que representa. Las entrevistas (realizadas entre julio y agosto del año 2018) fueron grabadas, alcanzando una hora de extensión en promedio. Luego de ser transcritas, fueron examinadas por medio del software ATLAS.ti (versión 15) en base a un análisis de contenido cualitativo, el cual permitió describir un corpus textual desarrollando un doble ejercicio de análisis deductivo e inductivo, para hacer emerger los significados latentes de las unidades de análisis.

En términos de las organizaciones humanitarias que operan en Chile, el contexto nacional replica la heterogeneidad de la esfera organizada descrita por estudios internacionales, con la Rahch como su micro-relato. Esta red surge a partir del terremoto que afectó al país en

26 GORDON, S., DONINI, A.: "Romancing principles and human rights: Are humanitarian principles salvageable?”. En: International Review of the Red Cross, Vol. 97, № (897898), 2015. p. 83. 
2010, siendo formada el 5 de diciembre de 2011 como una iniciativa de múltiples OSC que asumen "el compromiso de ser un aporte a la prevención, enfrentamiento y reparación de los daños humanos y materiales originados por desastres" ${ }^{27}$. Su principal objetivo corresponde a la promoción del fortalecimiento de capacidades, cooperación técnica y coordinación entre los organismos que componen la red, a fin de proporcionar una ayuda humanitaria eficiente y oportuna para lograr un Chile más seguro y resiliente ${ }^{28}$.

La Rahch agrupa organizaciones que comparten un fin humanitario, sus características son sumamente variadas. En términos comparativos, las organizaciones miembros incursionan en distintas áreas de intervención (voluntariado comunitario, facilitación de servicios de salud mental, búsqueda y rescate agreste, protección de derechos de la infancia, entre otras), y algunas incluyen más de un objeto de intervención en virtud de su carácter multimandato. Por otro lado, la Rahch congrega a organizaciones que presentan una larga trayectoria junto con otras cuya creación es considerablemente más reciente, incluye a organizaciones de raíz religiosa con otras laicas, y a organizaciones tanto nacionales como internacionales.

En la tabla 1 se presentan algunas características generales de las organizaciones abordadas, junto con un nombre en código para impedir su identificación y resguardar así la anonimidad de los datos. No obstante, se optó por hacer una excepción con la Cruz Roja Chilena, considerando su carácter de filial de la organización internacional fundadora del movimiento humanitario que ha perdurado -históricamente- como representante oficial de la acción humanitaria mundial, y que por ende, actúa como el organismo protector de los principios humanitarios.

27 RAHCH: Acta de Constitución de la Red de Ayuda Humanitaria Chilena Rahch, 2011a. p.1.

28 RAHCH: Marco Regulatorio, 2011b. p. 1. 
Tabla 1: Miembros de la Red Chilena de Ayuda Humanitaria (Rahch)

\begin{tabular}{|c|c|c|}
\hline Orientación & Origen & Código \\
\hline Laica & Nacional & OSC1 \\
\hline Religiosa & Internacional & OSC2 \\
\hline Laica & Nacional & OSC3 \\
\hline Laica & Internacional & Cruz Roja Chilena \\
\hline Laica & Internacional & OSC5 \\
\hline Laica & Nacional & OSC6 \\
\hline Religiosa & Internacional & OSC7 \\
\hline
\end{tabular}

Sobre el objeto a analizar (qué), se identificaron racionalidades (sentidos), prácticas y normas. Estos tres elementos analíticos son abordados en función de las fórmulas de armonización implicadas en la interacción de las organizaciones humanitarias con los principios humanitarios clásicos, lo cual es descrito a continuación.

\section{PRINCIPIO DE HUMANIDAD Y SINCRETISMO NORMATIVO.}

La dispersión del humanitarismo en múltiples racionalidades ha sido tematizada como un obstáculo para la colaboración inter-organizacional, puesto que la pluralidad de valores éticos, enfoques de intervención y prioridades operativas pueden resultar incompatibles entre $s^{29}$. No obstante, aunque los sentidos que las organizaciones de la Rahch construyen del imperativo humanitario son coincidentes en los aspectos centrales del principio (aliviar el sufrimiento y proteger la vida humana), este principio no es interpretado de modo literal, siendo -más bien- adaptado según el propio marco comprensivo de cada organización. Puesto que su interpretación articula elementos provenientes del ámbito humanitario con elementos extra-humanitarios propios de cada cultura organizacional, es factible hablar de una suerte de sincretismo normativo.

Proveniente del campo antropológico, el concepto de sincretismo es definido como un proceso mediante el cual se articulan de forma selectiva elementos provenientes de distintas matrices culturales, lo cual da lugar a objetos simbólicos que representan rasgos propios de éstas. En el campo

29 LABBÉ, J., DAUDIN, P.: “Applying the humanitarian principles: Reflecting on the experience of the International Committee of the Red Cross". En: International Review of the Red Cross, Vol. 97, N897/898, 2016. p. 202. 
jurídico, el uso más difundido del concepto de sincretismo proviene de Kelsen $^{30}$, que denomina como "sincretismo metodológico" al ejercicio de incorporar métodos propios de otras disciplinas al estudio del derecho. El concepto de sincretismo normativo aquí propuesto no guarda relación con la integración de disciplinares para contribuir a la ciencia jurídica -como plantea Kelsen ${ }^{31}$-, sino a cómo la armonización normativa es facilitada en el plano del sentido a través de un bricolaje de distintas culturas organizacionales.

A nivel de las jurisdicciones político-jurídicas nacionales, autores como De Carvalho describen cómo la imbricación de diferentes culturas jurídicas da como resultado nuevas estructuras normativas, lo cual puede ser descrito a través del concepto de sincretismo:

"Legal syncretism is present, at a lesser or greater extent, in all States, above all in those originating from decolonization processes and in those, wich at the pretext of adopting a modernizing legal policy, have "Westernized" their law"." 32

Asimismo, en el ámbito de las estructuras jurídicas de raíz religiosa, Salaymeh examina cómo la ley islámica se fue desarrollando en un contexto de pluralismo jurídico caracterizado por la interacción de tradiciones legales islámicas con otras no-islámicas:

"The recycled artwork of Islamic law transformed and combined may sources to create inventive compositions [...] much law was simultaneously Islamic and tribal, Jewish, or customary. Rather than labelling a law's presumed "identity", each of the three case studies in this book illustrated instead the recycled art of Islamic jurisprudence [...] These case studies presented glimpses of Islamic legal syncretism." ${ }^{\text {33 }}$

Considerando la observación de procesos de sincretismo normativo entre distintas culturas jurídicas a escala nacional y también en el plano religioso, el caso de estudio analizado en la presente investigación permite observar formas de sincretismo normativo en el contexto de los regímenes sectoriales de derecho. Más específicamente, se observa que el imperativo

30 KELSEN, H.: Acerca de las fronteras entre el método jurídico y el sociológico. En: Correas, O. (Ed.): El Otro Kelsen. Universidad Autónoma de México, México D. F., 1989. p. 285.

31 Ídem.

32 DE CARVALHO, E.: Semiotics of international law: Trade and translation. Springer Science \& Business Media, 2010. p. 64.

33 SALAYMEH, L.: The Beginnings of Islamic Law. Late Antique Islamicate Legal Traditions. Cambridge University Press, 2018. p. 198. 
humanitario es mezclado con fragmentos de cada cultura organizacional: la dignidad humana (OSC1), el amor cristiano (OSC2 y OSC7), la fraternidad (OSC5), o -incluso- visiones operativas como el resguardo de la salud mental (OSC6). A modo de ejemplo, la OSC1 corresponde a una organización que adopta a los derechos humanos como núcleo de sus intervenciones. Al ser consultada por los principios éticos que guían esa organización, la persona responsable indicó:

"Todos los que emanan directamente desde los derechos humanos. Sus cuatro principios fundamentales: universalidad, responsabilidad, indivisibilidad y participación [...] Hay una complementariedad con los cuatro principios humanitarios, porque como todos se construyen en torno al concepto de la dignidad, los principios de una u otra manera responden también de alguna manera a esos cuatro principios de los derechos humanos en términos más generales, por ejemplo esto de que la ayuda tiene que ser proporcional a las necesidades de las personas. Esa noción, por ejemplo, ese es el principio de no-discriminación que es exactamente el mismo principio de la universalidad, que los derechos son para todos de la misma manera. Entonces eso es, tal cual, la imparcialidad que es lo contrario a la no-discriminación." (OSC1)

Otro ejemplo lo representa la OSC5, que pone en primer plano la necesidad de promover la fraternidad y la convivencia, valores rectores de la institución, como ejes de la ayuda humanitaria. Respecto a sus principios y cómo se relacionan con el principio de humanidad, la persona entrevistada señala:

"[El primer principio] habla de la posibilidad de vivir de manera más fraternal con los demás seres humanos. El segundo principio nos inspira precisamente a estudiar también de manera comparada distintas ciencias, artes, filosofía. Y el tercero tiene que ver también con el desarrollar las potencialidades del ser humano [...] Y ahí está la ayuda humanitaria, que siempre va a haber gente más vulnerable, gente que necesite la ayuda, y si tú estás un poquito más preparado en ese evento, es una cuestión humana ponerse a disposición." (OSC5)

Por último, resaltan los ejemplos de las OSC2 y OSC7, organizaciones que interpretan el imperativo humanitario bajo los códigos simbólicos cristianos, articulando la ética humanitaria con su base ética y ontológica de corte religioso. En el caso de la OSC2, la persona entrevistada menciona:

"El piso siempre para nosotros van a ser los principios humanitarios. Agregamos como algunos principios que tienen ver más con nuestra 
naturaleza como ONG, que tienen su razón de ser en la Iglesia, por lo tanto nuestro llamado de acción y nuestra cosmovisión al respecto de cómo es que miramos la vida está fundamentada en que nosotros creemos que existe un Dios que es un Dios de amor, y que en base al ejemplo de Jesús, fue lo primero que él hizo, cuando se enfrentó a la humanidad fue suplir las primeras necesidades [...] ese es como nuestro llamado de acción, es un llamado de acción en base al amor, en base a la justicia, en base a la compasión, en saber que hay gente que está sufriendo, y que tú no puedes quedarte sentado." (OSC2)

La armonización del principio humanitario a escala inter-organizacional también se ve facilitada por la transversal adhesión al enfoque de derechos humanos y al de desarrollo humano por parte de las organizaciones examinadas. En virtud de lo anterior, el sentido que se forman del principio humanitario las organizaciones examinadas se desprende tanto de la misma racionalidad humanitaria, como del sentido y la praxis asociada a sus cosmovisiones y acciones no-humanitarias. Esto se corresponde con el carácter multimandato de dichas organizaciones, naturaleza atribuible incluso a la Cruz Roja Chilena. En efecto, esta institución ha incorporado de modo notable el paradigma de desarrollo humano en su horizonte ético y operativo, lo cual se observa normativamente incluso en su misión:

"Mitigar el sufrimiento humano y mejorar la calidad de vida de las personas, convirtiéndolas en los principales actores en la protección y recuperación de su seguridad y de su salud" 34

En este punto se observa un rasgo en común a las organizaciones examinadas, que de una u otra manera incorporan el enfoque de desarrollo humano en sus marcos éticos. En palabras de Vera (2016), la articulación de la acción humanitaria con la promoción del desarrollo humano destaca como un rasgo cultural propio de la ayuda humanitaria en Chile, sector que intensificó sus esfuerzos por superar el asistencialismo y promover la autonomía de los damnificados posteriormente a la crisis que provocó el terremoto ocurrido el 27 de febrero de 2010.

En el plano internacional, existe evidencia de intentos en otros países por recurrir al sincretismo normativo como medio para facilitar la implementación local de los principios humanitarios globales. Por ejemplo, Gordon y Donini ${ }^{35}$ plantean que en el sudeste asiático la neutralidad

34 CRUZ ROJA CHILENA: Plan Estratégico 2019-2022, 2018. p. 3.

35 GORDON, S., DONINI, A.: "Romancing principles and human rights: Are humanitarian principles salvageable?". En: International Review of the Red Cross, Vol. 97, № (897898), 2015. p. 101. 
y la independencia son secundarios al principio de no-interferencia, y específicamente en Japón el Estado es comprendido como el principal proveedor de asistencia humanitaria, además de figurar como el más adecuado y legítimo.

Justamente como método para intentar revertir la dispersión normativa, desde comienzo de la década del 2000 el ICRC ha emprendido conversaciones con sus filiales nacionales y con organizaciones humanitarias religiosas para generar convergencia entre "los principios, y otros marcos de referencia culturales, religiosos o filosóficos:" ${ }^{36}$. Al respecto, destaca el ejemplo de la Cruz Roja China por compatibilizar los principios humanitarios con aspectos culturales propios de dicho país. Además de adscribir a los siete principios del Movimiento de la Cruz Roja y de la Media Luna Roja, la Cruz Roja China incluyó dos "espíritus" (principios) adicionales que representan sus valores idiosincráticos: fraternidad y dedicación ${ }^{37}$. De este modo, la adopción de los principios humanitarios globales no se limita a la mera traslación forzosa de sus disposiciones, sino a la búsqueda de puntos de compatibilidad normativa mediante los cuales las normas cosmopolitas son adaptadas de modo congruente con la identidad cultural de cada organización (y país al cual pertenece).

\section{INTERPRETACIÓN MINIMALISTA DE LA INDEPENDENCIA FINANCIERA.}

En Chile, desde mediados de la década de 1980 hasta la actualidad, factores como el repliegue del Estado junto con la formación de una ciudadanía más participativa y con un rango más amplio de intereses han estimulado una considerable proliferación y diversificación de las $\mathrm{OSC}^{38}$, lo que desde 1990 en adelante ha venido aparejado con una creciente demanda de financiamiento ${ }^{39}$. Como medida para -entre otras cosas- mejorar los criterios de elegibilidad y consolidar las fuentes de

36 LABBÉ, J., DAUDIN, P.: “Applying the humanitarian principles: Reflecting on the experience of the International Committee of the Red Cross". En: International Review of the Red Cross, Vol. 97, N897/898, 2016. p. 193 (Traducción propia).

37 La OSC2 ha realizado un ejercicio similar al de la Cruz Roja China, agregando el valor de la dignidad a su acción humanitaria.

38 IRARRÁZAVAL, I., STREETER, P.: Mapa de las Organizaciones de la Sociedad Civil 2020. Centro de Políticas Públicas UC, Fundación Chile+Hoy, Santiago, 2020. p.20.

39 SOTO, F., VIVEROS, F.: Las organizaciones de la sociedad civil desde su marco jurídico e institucional. Configurando un actor social. Centro de Políticas Públicas UC, Fundación Chile +Hoy, Santiago, 2016.p. 13. 
financiamiento estatal para la sociedad civil organizada, en 2011 se crea Fondo de Fortalecimiento de las Organizaciones de Interés Público a través de la Ley 20.500, el que no obstante se ha mostrado incapaz de resolver los problemas de sostenibilidad financiera de las $\mathrm{OSC}^{40}$.

De todos modos, los pagos gubernamentales continúan representando la principal forma de financiamiento para las OSC en el país, alcanzando el $49 \%$ del total si se considera además a los ingresos propios obtenidos por ventas de bienes y servicios (30\%) y a las donaciones filantrópicas $(21 \%)^{41}$. En esta línea, el entorno institucional en cual se desenvuelven las organizaciones corresponde a un factor crítico en las perspectivas y prácticas asumidas por éstas, pudiendo obstaculizar la adhesión y/o cumplimiento de algunos principios humanitarios. En el caso de la Rahch, los entrevistados refieren a la falta de apoyo por parte del Estado (en términos financieros y operacionales) como un problema que se interpone en el correcto despliegue de sus acciones humanitarias. A modo de ejemplo, al ser consultado sobre la frecuencia con la que se cumplen los principios por parte de las OSC humanitarias en Chile, el representante de la OSC3 Chile comenta:

"Sí, pero algunos no. El primero sí, el de humanidad. Lo que pasa es que esto está enfocado en grandes desastres. En grandes desastres sí, todos se cumplen. Pero en tiempos normales pasa eso de que las ONG no tienen buena situación económica, se van mejor a un municipio, buscan recursos en otro lado." (OSC3)

Las necesidades de financiamiento y la dependencia que mantienen con el Estado impactan en el sentido que las organizaciones examinadas atribuyen al principio de independencia financiera, en la medida que éste es adaptado por medio de un recurso de interpretación minimalista, siendo entendido de modo funcional a las circunstancias económicas en las que se encuentra el tercer sector. En concreto, si bien los representantes entrevistados aceptan el propósito de la independencia (es decir, impedir la corrupción de la ayuda humanitaria a manos de fuerzas externas) no sucede lo mismo con la forma en que el principio podría expresarse en la práctica. Más específicamente, la interpretación del principio lo flexibiliza

40 IRARRÁZAVAL, I., SAGREDO, M.P., LIZANA, M.: Un nuevo trato para las organizaciones de la sociedad civil. Desafios y propuestas. Centro de Políticas Públicas UC, Fundación Chile +Hoy, Santiago, 2019. p. 89.

41 Cifras elaboradas por la OSC Sociedad en Acción (Centro de Políticas Públicas de la Pontificia Universidad Católica de Chile y fundación Chile+Hoy), disponibles en su sitio web. Véase: https://www.sociedadenaccion.cl/estudios/osc-en-cifras/ 
endosándole una cláusula a su cumplimiento: sería aceptable recibir fondos de grupos con influencia e intereses políticos y económicos, siempre que su uso esté debidamente transparentado ${ }^{42}$. A modo de ejemplo, las personas entrevistadas señalan:

"Lo que pasa es que la plata siempre es de alguien, o sea si uno dijera "no vamos a recibir ni de estos, ni de estos, ni de estos", se quedaría sin plata digamos. Uno podría recibirla, pero hacerlo público, hacerlo transparente." (OSC5)

"En la misma lógica del financiamiento de las campañas políticas. $\mathrm{O}$ sea no veo por qué no debería aceptarlo, siempre y cuando efectivamente su donación no venga...claro, sea transparente, y no venga amarrando o no interfiera o no busque incidir en las áreas programáticas de la acción." (OSC1)

La necesidad de las OSC de ampliar las fuentes de donaciones asume un matiz neo-humanitario, lo cual se manifiesta en la relajación de los criterios para acceder al financiamiento, y refleja también un rasgo idiosincrático de concebir los principios humanitarios por parte de la sociedad civil organizada que opera en el país, moldeada en gran medida por las condiciones estructurales de la institucionalidad chilena.

En términos hipotéticos, esta interpretación laxa de la independencia financiera puede ser admitida como válida por el resto del campo humanitario, lo que reconocería implícitamente la flexibilidad semántica de la norma, o bien ser rechazada por la pérdida de autonomía que representa para las OSC. En este sentido, la ambigüedad interpretativa del principio denota tanto una ventaja como una desventaja, dado que si bien predispone $\mathrm{y}$ facilita su adhesión por parte de actores organizacionales locales (y con ello, la difusión y aceptación de los principios humanitarios), deja a los mismos sujetos de la regulación la tarea de delinear sus contornos prescriptivos e idear métodos para su aplicación, lo que puede dar lugar a una armonización que resulta válida solo en términos nominales.

Más precisamente, estas declaraciones pasan por alto las relaciones de reciprocidad que se construyen entre dos partes al aceptar recursos de manera aparentemente desinteresada, como lo ha desarrollado la antropología clásica y su consiguiente adaptación a los estudios sobre la conformación

42 A excepción de la Cruz Roja Chilena, que mantiene una interpretación estricta del principio. 
de redes clientelares en política ${ }^{43}$. Más específicamente, aceptar fondos de fuentes que concentran suficiente poder como para influenciar la dirección de las organizaciones puede resultar en formas de cooptación operativa. En sintonía con esta hipótesis, se ha descrito una situación de "captura" de las OSC por parte del Estado en Chile, dando lugar a una tercerización de la sociedad civil organizada al actuar como simples ejecutores de políticas y programas públicos ${ }^{44}$. Más aún, el hecho de que las OSC dependan del Estado para continuar operando puede impedir aplicar el principio de neutralidad en las controversias donde esté involucrado el Estado.

En definitiva, este recurso representa un riesgo como fórmula de armonización, en la medida que esta aceptación se logra al precio de permitir interpretaciones inconsistentes, lo que crea una consistencia interorganizacional de la ética humanitaria solo en términos formales (o aparentes), engendrando con ello potenciales colisiones en estado latente.

\section{ADOPCIÓN DE LOS PRINCIPIOS EN ESTRUCTURAS PERIFÉRICAS Y EQUIVALENTES OPERATIVOS.}

En relación al énfasis que ponen las organizaciones en los resultados de sus intervenciones, los discursos expresan de modo recurrente la necesidad de evitar malas prácticas, lo cual se ejemplifica en el imperativo de la "efectividad" pregonado por OSC6, en la necesidad de evitar crear "crisis dentro de las crisis" planteada por OSC5, o en un discurso que resalta la necesidad de actuar sin poner en riesgo a los mismos voluntarios o a los damnificados (OSC3). La relevancia que las OSC humanitarias adjudican a la eficiencia guarda relación con el potencial daño iatrogénico ${ }^{45}$ que puede generar su actuar en las comunidades receptoras, defecto que alcanzó especial visibilidad mundial con el genocidio de Ruanda en 1994, el cual dejó al descubierto altas tasas de mortalidad y la prolongación del conflicto producidas por una acción humanitaria carente de estándares y mecanismos de rendición de cuentas (accountability) ${ }^{46}$.

43 HOEVEL, C., MASCAREÑO, A.: "La emergencia de redes clientelares en América Latina: Una perspectiva teórica". En: Revista Mad, N³4, 2016. p. 39.

44 SOTO, F., VIVEROS, F.: Las organizaciones de la sociedad civil desde su marco jurídico e institucional. Configurando un actor social. Centro de Políticas Públicas UC, Fundación Chile +Hoy, Santiago, 2016. p. 9.

45 Es decir, el daño causado involuntariamente por una acción orientada a curar o sanar.

46 GORDON, S., DONINI, A.: "Romancing principles and human rights: Are humanitarian principles salvageable?”. En: International Review of the Red Cross, Vol. 97, N (897898), 2015. p. 105. 
Aunque se observa una considerable adhesión a los principios humanitarios en el plano del sentido, la mayoría de las organizaciones presenta una escasa o nula incorporación de los principios dentro de sus marcos normativos. Sin embargo, en la totalidad de los casos se señala que las acciones de despliegue humanitario se rigen por lo que determina el Manual Esfera. Esta realidad da cuenta que se privilegia la adopción de normas técnicas por sobre las éticas, lo cual corrobora un hallazgo de Vera, quien examinó el funcionamiento de diversas OSC humanitarias en la Región del Biobío, dando cuenta que "siendo que muchos [de los actores humanitarios] desconocen los principios específicos que distinguen a esta clase de ayuda, todos los consultados concuerdan con ellos y los mencionan -con sus palabras- como la base de su actuar." ${ }^{47}$

Más específicamente, el material analizado permite identificar dos tipos de argumentos para justificar la no incorporación de las normas humanitarias en el marco ético de las organizaciones:

a) Sus propios valores o principios son equivalentes a los principios humanitarios, por lo que al estar incluidos indirectamente no sería necesario adoptarlos de modo formal.

b) El mandato humanitario es solo uno de los múltiples imperativos que cumple la organización, razón por la cual no sería pertinente asumirlos como transversales al marco ético de la OSC.

Sobre el último argumento, hay organizaciones multimandato como OSC7 y OSC2 que si bien no han incorporado los principios en el centro o núcleo de su marco ético (es decir, en su misión, visión y valores), sí han incorporado algunos de ellos en estructuras normativas "periféricas", representadas por manuales, circulares informativas o protocolos propios de sus respectivas áreas de emergencias. Esta fórmula permite ubicar la ética humanitaria en un espacio normativo específico de la organización, de tal manera que se evita crear distorsiones en áreas -y mandatos- ajenos a la acción propiamente humanitaria.

Ahora bien, tanto la razón (a) como la (b) denotan una postura institucional que adhiere a los principios humanitarios desde el punto de vista del sentido que le atribuyen, pero lo descarta por razones prácticas. La reticencia a codificar de modo explícito estos principios en los marcos éticos de las organizaciones repercute en el modo a través del cual éstos

47 VERA, N.: Respuesta Humanitaria en Situaciones de Desastre. Universidad de Concepción, 2016. p. 29. 
son adoptados, difundidos, reflexionados y operacionalizados por parte de cada cultura organizacional. A su vez, su ausencia normativa influye en la formación de capacidades y producción de conocimiento de la organización, y dificulta o sencillamente imposibilita auditarlos de ser necesario.

Además de la incorporación algunos principios humanitarios exclusivamente en los lineamientos del área de emergencias de las organizaciones, otro ejemplo de armonización haciendo uso de estructuras normativas periféricas consiste en la adopción de metodologías para ordenar la entrega de la asistencia humanitaria exclusivamente en base al grado de necesidad de las personas, lo cual satisface el criterio de proporcionalidad ${ }^{48}$ del principio de imparcialidad aunque éste no se encuentre incluido en el marco ético oficial de la organización. Ejemplo de este equivalente operativo consiste en la metodología Triage START (Simple Triage And Rapid Treatment) utilizada por la OSC3, la cual clasifica el estado de salud y vulnerabilidad de las víctimas en cuatro niveles, para priorizar la intervención según la gravedad y facilidad de acceso al lugar donde se ubican las personas definiendo un tiempo máximo de espera para cada perfil.

Esta fórmula de armonización, claro está, respeta los límites éticos del sentido que cada organización atribuye a la acción humanitaria, por lo que resulta factible emplearla siempre que el principio en cuestión sea compatible con sus respectivas racionalidades. Esta incongruencia entre las racionalidades y los principios humanitarios se observa, por ejemplo, en que tanto OSC2 como OSC7 eluden incorporar el principio de neutralidad y la dimensión política del principio de independencia ${ }^{49}$.

48 Es decir, se debe aliviar el sufrimiento de los individuos de manera proporcional al grado de su sufrimiento, dando prioridad acorde a su grado de urgencia.

49 La independencia política se entiende como autonomía en la toma de decisiones, sin la influencia de grupos como partidos políticos, el Estado o instituciones de orden religioso. Ahora bien, El rechazo de la neutralidad se explica porque ambas organizaciones se encuentran guiadas por el concepto cristiano de justicia social, el cual puede inducir la participación de la organización en controversias, asumiendo una postura partidaria a favor de personas o grupos oprimidos. Por otra parte, la omisión del reconocimiento de la independencia política es coherente con la incidencia eclesiástica en el gobierno corporativo de cada institución, implicando que su conducción se pueda ver influenciada por las prioridades que cada iglesia determina. 


\section{VENTAJAS Y DESVENTAJAS DE LA ARMONIZACIÓN NORMATIVA.}

Lo descrito hasta el momento da cuenta sobre las múltiples y diversas fórmulas a las que recurren las organizaciones humanitarias para armonizar su sentido, normas y prácticas con los principios humanitarios clásicos. Sin embargo, la inconsistencia entre estos tres elementos puede ocultarse tras una aparente armonización normativa. Para comprender de forma esquemática la distinción entre la expresión normativa de los principios humanitarios y su resultado práctico, resulta útil la clasificación que formula Lerner $^{50}$ :

Tabla 2: Cuatro casos de armonización

\begin{tabular}{|c|c|}
\hline I. Normativa similar - mismo resultado & II. Normativa diferente - distinto resultado \\
\hline III. Normativa diferente - mismo resultado & IV. Normativa similar - distinto resultado \\
\hline
\end{tabular}

En relación a las fórmulas de armonización descritas, el sincretismo normativo -aplicado principalmente al principio de humanidad- sería identificable con el cuadrante II (norma diferente - distinto resultado), en la medida que los principios de distintos sistemas de pensamiento son adaptados, articulados e integrados con la ética humanitaria, lo cual permitiría producir efectos equivalentes en el plano operativo. Claro está, este sincretismo se facilita en algunos casos gracias la raíz cristiana del humanitarismo moderno ${ }^{51}$, y en otros al hecho de que el sistema universal de los derechos humanos surge posteriormente al movimiento humanitario ${ }^{52}$, lo que indudablemente influyó en su diseño teórico-moral. De este modo, tanto las OSC eclesiásticas como las que surgen para promover y defender los derechos humanos se benefician de la imbricación que existe entre el humanitarismo y otros sistemas de pensamiento, ya sea porque el movimiento humanitario emerge desde su base o porque éste sirve de base para la emergencia de matrices epistémicas posteriores.

50 LERNER, P.: "Sobre armonización, derecho comparado y la relación entre ambos". En: Boletín mexicano de derecho comparado, Vol. 37, N¹11, 2004. p. 942.

51 KRAFT, K.: "Faith and impartiality in humanitarian response: Lessons from Lebanese evangelical churches providing food aid". En: International Review of the Red Cross, Vol. 97, $\mathrm{N}^{\circ} 897-898,2016$. p. 396.

52 LEEBAW, B.: "The politics of impartial activism: humanitarianism and human rights". Perspectives on Politics, Vol. 5, N², 2007. p. 224. 
La flexibilidad interpretativa del principio de independencia financiera, por otra parte, se identificaría con la situación descrita por el cuadrante IV (norma similar - distinto resultado). Lerner ${ }^{53}$ señala que la discrepancia entre forma y resultado puede responder a dos razones: los sistemas jurídicos reconoce la misma norma pero le confieren un significado distinto, o las normas son equivalentes pero su aplicación es distinta. En este caso, la independencia financiera justamente es interpretada de un modo particular por las OSCs abordadas, las cuales operan en un entorno institucional caracterizado por la dificultad para conseguir recursos que permitan la sostenibilidad económica. En la práctica, la mayoría de las OSCs no se dedican exclusivamente a la acción humanitaria -de forma consistente con su naturaleza multimandato-, por lo que reciben financiamiento simultáneamente de distintas fuentes para distintas áreas de intervención (por ejemplo, recibir fondos para ejecutar unas políticas ministeriales o municipales en unas áreas, mientras financia otras con donaciones filantrópicas o con ventas de servicios.

Con respecto a la incorporación de los principios humanitarios en estructuras periféricas y equivalentes operativos (principalmente en relación al principio de imparcialidad), las fórmulas en este caso están orientadas a ubicar las normas humanitarias en un espacio separado del núcleo ético de la organización, entendiendo que su carácter multimandato implica la fragmentación de la organización en áreas con objetivos y enfoques claramente delimitados. Por lo tanto, esta fórmula es identificable a la situación descrita en el cuadrante I (norma similar - mismo resultado).

Por último, con respecto al principio de neutralidad, dos organizaciones (OSC1 y OSC2) lo rechazan explícitamente por ser incompatible con la búsqueda de la justicia basada en los derechos humanos y con los preceptos cristianos del bien común. Más aún, la única OSC que incorpora la neutralidad en su marco normativo corresponde a la Cruz Roja Chilena. Sin embargo, las organizaciones que renuncian a la neutralidad han participado en actividades de incidencia en políticas públicas y han recurrido a formas defensa jurídica dentro de un marco legalista y discreto, lo cual coincide con el método a través del cual la Cruz Roja Chilena cumple con el principio de neutralidad. Así, aunque divergen en el sentido que atribuyen al principio, coinciden en sus prácticas. Por lo tanto, se observa un caso identificable

53 LERNER, P.: "Sobre armonización, derecho comparado y la relación entre ambos". En: Boletín mexicano de derecho comparado, Vol. 37, N¹11, 2004. p. 943. 
con el cuadrante III en el esquema de Lerner ${ }^{54}$, dado que si bien la norma es distinta el resultado es el mismo.

En relación con lo anterior, la defensa de los principios de independencia $\mathrm{y}$-por sobretodo- de neutralidad ha sido justificada en base a razones más teleológicas que deontológicas. Como plantean Özerdem y Rufini ${ }^{55}$, son dos los escenarios a los que se enfrentan las OSC humanitarias dependiendo si aplican o no los principios de neutralidad.

En primer lugar, si las agencias humanitarias observan abusos a los derechos humanos por parte de alguna de las partes beligerantes, y optan por denunciar públicamente a los perpetradores (es decir, rechazar la neutralidad), se corre el riesgo de ser expulsados de la zona de conflicto y con ello verse incapaces de satisfacer directamente el imperativo humanitario en el largo plazo, lo que incluye tanto esa como futuras emergencias. $\mathrm{Abiew}^{56} \mathrm{y} \mathrm{Mills}^{57}$ refuerzan esta idea, asegurando que las instituciones que no adoptan el principio de neutralidad suelen ser objeto de ataques por parte de las partes en conflicto, o bien, simplemente se les niega el acceso las zonas en emergencia. En segundo lugar, las agencias humanitarias pueden ignorar las vulneraciones a los derechos humanos presenciadas para asegurar la continuación de su actividad territorial, manteniendo así la neutralidad. Sin embargo, pueden ser acusados de asumir complicidad con los perpetradores, como le ha criticado históricamente Médicos Sin Fronteras a la Cruz Roja ${ }^{58}$.

Mientras que la primera postura es representada por las OSCs "neohumanitarias", la segunda refleja fielmente la visión ortodoxa del ICRC. La neutralidad, por lo tanto, corresponde a un método práctico para construir confianza con los demás stakeholders del régimen humanitario, lo que contribuye a asegurar la aceptación de la ayuda en situaciones de conflicto armado. Como plantean Labbé y Daudin, las colisiones entre principios generalmente se traducen en una contraposición de los objetivos de corto y

54 Ibídem, p. 942.

55 ÖZERDEM, A., RUFINI, G.: Humanitarianism and the Principles of Humanitarian Action in Post-Cold War Context. En: Barakat, S.: After the conflict: reconstruction and development in the aftermath of Conflict. IB Tauris, London, 2005. p. 58.

56 ABIEW, F. K.: "Humanitarian action under fire: Reflections on the role of NGOs in conflict and post-conflict situations”. En: International Peacekeeping, Vol. 19, ํํㄹ, 2012. p. 208.

57 MILLS, K.: "Neo-humanitarianism: the role of international humanitarian norms and organizations in contemporary conflict". En: Global Governance, Vol. 11, 2005. p. 171.

58 BRAUMAN, R.: "Médecins Sans Frontières and the ICRC: matters of principle". En: International Review of the Red Cross, Vol. 94, N888, 2012. p. 1527. 
largo plazo: “...entre salvar vidas hoy con pocos compromisos, y mantener la capacidad de la organización para salvar vidas mañana al mantenerse consistente y perseverando en la confianza de todos" ${ }^{59}$. En otras palabras, la renuncia a labores de incidencia política y denuncia por parte del humanitarismo clásico privilegia una postura reactiva, que proteger vidas $\mathrm{y}$ alivia en lo inmediato el sufrimiento humano. El neo-humanitarismo, por su parte, alcanza este objetivo trabajando ex-ante y ex-post, como forma de extender la acción humanitaria a otras fases del continuo.

Todo lo anterior permite argumentar que no necesariamente es preferible la armonización de principios humanitarios a su dispersión, generando rendimientos diferenciales. Más explícitamente, la armonización de principios humanitarios dota de mayor cohesión a las organizaciones involucradas, pudiendo generar efectos emergentes al actuar como bloque en actividades como la incidencia en políticas públicas. Asimismo, se reduce el riesgo de intromisión de prejuicios personales o intereses económicos/ políticos extra-humanitarios. Sin embargo, la existencia de organizaciones humanitarias neutrales y no-neutrales genera efectos agregados que beneficia al sistema humanitario en su conjunto: las primeras cuentan con una mayor capacidad para ser aceptadas en situaciones de despliegue, mientras que las segunda cumplen en mayor medida una función defensora de los derechos humanos.

Sin embargo, no están claros los resultados reales de esta división del trabajo humanitario, puesto que esta compatibilidad se produce solo a escala sistémica. En el nivel intra-organizacional, por ejemplo, no es posible concebir una organización que se defina simultáneamente como a favor y en contra de la neutralidad, por lo que no les resulta posible coordinarse. A nivel inter-organizacional, ambas posturas interactúan y devienen en colisiones que, a su vez, producen tensiones políticas entre las distintas organizaciones.

\section{CONCLUSIONES.}

La Rahch emerge como un caleidoscopio de realidades que replica la heterogénea esfera organizada descrita a nivel internacional, cual microrelato de sus diversas tensiones y complementariedades. Con excepción

59 LABBÉ, J. DAUDIN, P.: “Applying the humanitarian principles: Reflecting on the experience of the International Committee of the Red Cross". En: International Review of the Red Cross, Vol. 97, N897/898, 2016. p. 200. 
de la Cruz Roja Chilena, la ética que inspira a las organizaciones que componen la Rahch se asocia con lo que la literatura describe como neohumanitarismo, esto es, la interrelación del imperativo humanitario con los preceptos de los enfoques de derechos humanos y de desarrollo humano, aunque el balance de estos mandatos varía de organización en organización.

Aunque cada OSC construye un sentido sobre la ética humanitaria consistente con sus propios valores, misión, visión, $\mathrm{y}$, particularmente, con su ámbito de intervención, se evidencian compatibilidades dentro y entre las organizaciones respecto al principio de humanidad, ligadas a la postura teleológica que comparten transversalmente las entidades miembros, junto con la adopción intensiva el enfoque de desarrollo humano y en menor medida el de derechos humanos. En este sentido, fue posible identificar formas de sincretismo normativo en la adopción del principio humanitario $\mathrm{y}$ del principio de independencia financiera, los cuales son interpretados y adecuados a los marcos comprensivos, orientaciones prácticas y necesidades de cada organización, gracias a su laxitud semántica y flexibilidad procedimental. Además, se identificó una convergencia respecto a los principios de imparcialidad y de independencia financiera, aunque en este último caso el principio se flexibiliza, considerando las necesidades económicas de las organizaciones. De igual modo, la incorporación de los principios en estructuras periféricas y mediante equivalentes operativos representa un modo de adhesión a los principios que permite mantener intacto el núcleo ético de las organizaciones en cuestión, lo cual resulta congruente con su naturaleza multimandato.

Con respecto a la neutralidad, aquellas organizaciones que rechazan este principio han participado en actividades de incidencia en políticas públicas y han recurrido a formas defensa jurídica dentro de un marco legalista y discreto, lo cual coincide con el método a través del cual la Cruz Roja Chilena cumple con el principio de neutralidad. Así, aunque divergen en el sentido que atribuyen al principio, coinciden en sus prácticas. A nivel sistémico, la relación de las organizaciones neutrales y las no-neutrales no es calificable a priori como compatible o incompatible. Más bien, se sostiene que si bien las organizaciones neutrales y no-neutrales colisionan el plano del sentido, pueden ser complementarias en el ámbito de las prácticas. No obstante, no es posible generar formas de coordinación el plano del sentido o de las normas entre ambos tipos de acciones, por lo que su complementación ocurre más bien de manera espontánea. 
La discrepancia entre las posturas éticas de las OSC abordadas, es reflejo de una tensión global entre distintas formas de entender el humanitarismo. Claro está, las adaptaciones locales de los principios no necesariamente pueden ser aceptadas por el resto de la comunidad humanitaria, lo que como parte de las dinámicas de reconocimiento mutuo entre el nivel global y el local, implicaría su revisión por parte de otras jurisdicciones territoriales, organizaciones transnacionales y organismos internacionales.

\section{BIBLIOGRAFÍA}

ABIEW, F. K.: "Humanitarian action under fire: Reflections on the role of NGOs in conflict and post-conflict situations". En: International Peacekeeping, Vol. 19, N², 2012.

BENAVIDES, L.: El régimen internacional del "nuevo humanitarismo". Disertación doctoral, Universidad Autónoma de Madrid, Grupo de Estudios de Relaciones Internacionales (GERI), 2009.

BRAUMAN, R.: "Médecins Sans Frontières and the ICRC: matters of principle". En: International Review of the Red Cross, Vol. 94, $\mathrm{N}^{\circ} 888$, 2012.

CHS ALLIANCE, GROUP URD, PROYECTO ESFERA: La Norma Humanitaria Esencial en materia de calidad y rendición de cuentas (Core Humanitarian Standard), 2015.

CRUZ ROJA CHILENA: Plan Estratégico 2019-2022, 2018.

DE CARVALHO, E.: Semiotics of international law: Trade and translation. Springer Science \& Business Media, 2010.

FOX, F.: "New humanitarianism: does it provide a moral banner for the 21st century?". En: Disasters, Vol. 25, Nº4, 2001.

GORDON, S., DONINI, A.: "Romancing principles and human rights: Are humanitarian principles salvageable?". En: International Review of the Red Cross, Vol. 97, № (897-898), 2015.

HOEVEL, C., MASCAREÑO, A.: "La emergencia de redes clientelares en América Latina: Una perspectiva teórica”. En: Revista Mad, №34, 2016. 
IRARRÁZAVAL, I., STREETER, P.: Mapa de las Organizaciones de la Sociedad Civil 2020. Santiago, Centro de Políticas Públicas UC, Fundación Chile+Hoy, 2020.

IRARRÁZAVAL, I., SAGREDO, M.P., LIZANA, M.: Un nuevo trato para las organizaciones de la sociedad civil. Desafios y propuestas. Santiago, Centro de Políticas Públicas UC, Fundación Chile +Hoy, 2019.

KELSEN, H.: Acerca de las fronteras entre el método jurídico y el sociológico. En: Correas, O. (Ed.): El Otro Kelsen. Universidad Autónoma de México, México D. F., 1989.

KRAFT, K.: "Faith and impartiality in humanitarian response: Lessons from Lebanese evangelical churches providing food aid". En: International Review of the Red Cross, Vol. 97, N897-898, 2016.

LABBÉ, J., DAUDIN, P.: “Applying the humanitarian principles: Reflecting on the experience of the International Committee of the Red Cross". En: International Review of the Red Cross, Vol. 97, Nº897/898, 2016.

LEEBAW, B.: "The politics of impartial activism: humanitarianism and human rights". Perspectives on Politics, Vol. 5, N², 2007.

LERNER, P.: "Sobre armonización, derecho comparado y la relación entre ambos". En: Boletín mexicano de derecho comparado, Vol. 37, №111, 2004.

MILLS, K.: "Neo-humanitarianism: the role of international humanitarian norms and organizations in contemporary conflict". En: Global Governance, Vol. 11, 2005.

VERA, N.: Respuesta Humanitaria en Situaciones de Desastre. Universidad de Concepción, 2016.

ÖZERDEM, A., RUFINI, G.: Humanitarianism and the Principles of Humanitarian Action in Post-Cold War Context. En; Barakat, S.: After the conflict: reconstruction and development in the aftermath of Conflict. IB Tauris, London, 2005.

PICTET, J.: The Fundamental Principles of the Red Cross: Commentary By Jean Pictet. International Federation of Red Cross and Red Crescent Societies, 1979.

RAHCH: Acta de Constitución de la Red de Ayuda Humanitaria Chilena Rahch, 2011a. 
RAHCH: Marco Regulatorio, 2011b.

SALAYMEH, L.: The Beginnings of Islamic Law. Late Antique Islamicate Legal Traditions. Cambridge University Press, 2018.

SALEK, L. V.: "Faith inspiration in a secular world: An Islamic perspective on humanitarian principles". En: International Review of the Red Cross, Vol. 97, Nº897-898, 2015.

SCHENKENBERG, E.: "Coming clean on neutrality and independence: The need to assess the application of humanitarian principles". En: International Review of the Red Cross, Vol. 97, N897-898, 2016.

SOTO, F., VIVEROS, F.: Las organizaciones de la sociedad civil desde su marco jurídico e institucional. Configurando un actor social. Centro de Políticas Públicas UC, Fundación Chile +Hoy, 2016. 